Advances in Dynamical Systems and Applications (ADSA).

ISSN 0973-5321, Volume 15, Number 2, (2020) pp. 143-151

(C) Research India Publications

https://dx.doi.org/10.37622/ADSA/15.2.2020.143-151

\title{
A Topological Path to the Collatz Conjecture: A historical point of view
}

\author{
Fernando Mejias ${ }^{* 1}$, Jorge Vielma ${ }^{2}$ and Ángel Guale ${ }^{3}$ \\ 1,2,3 Professor of Mathematics, ESPOL Polytechnic University, Escuela Superior \\ Politécnica del Litoral, Facultad de Ciencias Naturales y Matemíicas, Campus \\ Gustavo Galindo Km. 30.5 Vía Perimetral, P.O. Box 09-01-5863, Guayaquil, Ecuador.
}

\begin{abstract}
In this paper a recent topological approach to the Collatz conjecture is shown. Also known as "the $3 x+1$ problem", the Collatz conjecture establishes that for every natural number $n$, there exists a natural number $r$ such that the $r$-fold composite $\kappa^{r}(n)=1$, where $\kappa: \mathbb{N} \rightarrow \mathbb{N}$ is the function defined as $x / 2$ if $x$ is even and as $3 x+1$ if $x$ is odd. This map $\kappa$ induces the so called primal topology $\tau_{\kappa}$ on $\mathbb{N}$. We prove that the space $\left(\mathbb{N}, \tau_{\kappa}\right)$ is path-connected and compact if and only if the Collatz conjecture is true.
\end{abstract}

Keywords: Collatz conjecture, primal space, Alexandroff space.

2010 Mathematics Subject Classification: 53A03, 06B30, 54F05, 54F65.

\section{INTRODUCTION}

The Collatz conjecture was formulated as an open problem in the first half of the 20th century, and since those days it has become a sort of a very special landmark in the world of mathematics.

Many reports about different attempts to approach the problem are available, especially the work by Lagarias [6] shows a wide range of accounts of such works. In this paper we describe the path traced during an exploration trip from the realm of topology.

\footnotetext{
*(Corresponding author)
} 
The Collatz conjecture is based on has been built around the map $\kappa: \mathbb{N} \rightarrow \mathbb{N}$ defined as $x / 2$ if $x$ is even and $3 x+1$ if $x$ is odd, and the compositions of the form $\kappa \circ \cdots \circ \kappa(x)$ for some natural numbers $x$, arriving always to 1 , and there they start moving in circles (or cycles) first to 4, then to 2, and so on. This is essentially the Collatz conjecture: For any $x \in \mathbb{N}$ there exists an $m \in \mathbb{N}$ such that the $m$-fold composite $\kappa \circ \cdots \circ \kappa(x)=1$ and the only cycle is $\{1,2,4\}$.

So far, every computation of this kind has always eventually arrived to 1, among them Harold Scott MacDonald Coxeter, Shizuo Kakutani, and Stanisław Marcin Ulam. Two more generic names are frequently used: "the Syracuse problem" and "the $3 x+1$ problem." The most common named associated to the problem is after the German mathematician Lothar Collatz who seems to have circulated the problem in 1950, at the International Congress of Mathematics in Cambridge, Massachusetts, and presumably knew about it since 1937, see Lagarias [6]. Thus several attempts to solve the problem have been done by many generations of mathematician, for some more recent insights see [8].

In this work we take an approach to the problem from a topological point of view, starting with the observation that the map $\kappa$ induces a topology $\tau_{\kappa}$ on the set $\mathbb{N}$ of the natural numbers and by using such structure it has been proved that the conjecture is true if and only if the topological space $\left(\mathbb{N}, \tau_{\kappa}\right)$ is connected and compact; moreover we show that all points of the space $\left(\mathbb{N}, \tau_{\kappa}\right)$ can be connected by a loop.

In order to complete this program some concepts from algebra are necessary, and by working on those lines Vielma and Guale [9] show that the Collatz conjecture is equivalent to the fact that the space $\left(\mathbb{N}, \tau_{\kappa}\right)$ has the structure of a local semiring. The idea of using such an algebraic structure for topological spaces is based on the work by Di Maio [2] and a paper by Echi and Turki [4].

\section{BASICS OF THE COLLATZ CONJECTURE}

In order to understand the Collatz conjecture we set some conventions on notation: Let $\mathbb{Z}^{+}$denote the set of nonnegative integers. Given a map $f: X \rightarrow X$, and a nonnegative integer $r$, we denote by $f^{r}$ the identity map on $X$ if $r=0, f$ itself if $r=1$, and the $r$-fold composite $f \circ \cdots \circ f$ for $r>1$.

Definition. Given a set $X \neq \emptyset$ and $x \in X$, the orbit of $x$ is the set $\Omega_{x, f}=\left\{f^{r}(x): r \in\right.$ $\left.\mathbb{Z}^{+}\right\}$. The orbit of $\Omega_{x, f}$ is said to be periodic if there exists a natural number $r$ such that $f^{r}(x)=x$. 
Now consider the Collatz map $\kappa: \mathbb{N} \rightarrow \mathbb{N}$, which is defined by

$$
\kappa(n)= \begin{cases}n / 2 & \text { if } n \text { is even } \\ 3 n+1 & \text { if } n \text { is odd }\end{cases}
$$

In the following we use $\Omega_{n}$ instead of $\Omega_{n, \kappa}$. It is easy to verify that $\Omega_{1}=\{1,2,4\}$ is a periodic orbit and that $1 \in \Omega_{p}$ for all $p=2^{m}, m \in \mathbb{N}$.

The Collatz conjecture can be stated as follows: For any $n \in \mathbb{N}$ we have that $1 \in \Omega_{n}$ and the only periodic orbit is $\Omega_{1}$.

\section{PRIMAL TOPOLOGIES}

Some different techniques have been developed to attack the Collatz conjecture from different fields, for example Bernstein and Lagarias [1] apply the concept of conjugacy to approach this problem from a topological point of view. In this section we set the basis to take another topological approach, and the main idea is investigate a topology induced on the set $\mathbb{N}$ of the natural numbers by the Collatz map $\kappa$ which is derived by applying the following result.

Proposition 1. Given a nonempty set $X$ and a function $f: X \rightarrow X$, let us consider the collection $\tau_{f}$ of all sets $O \subset X$ such that $O \in \tau_{f}$ if and only if $f^{-1}(O) \subset O$. Then $\tau_{f}$ is a topology for $X$.

Proof. It is obvious that $\emptyset$ and $X$ belong to $\tau_{f}$. Suppose that there exists a set of indexes $A$ and that $V=\left\{V_{\alpha}: \alpha \in A\right\}$ is an arbitrary collection of elements of $\tau_{f}$, then $f^{-1}\left(V_{\alpha}\right) \subset V_{\alpha}$ for all $\alpha$, therefore

$$
f^{-1}\left(\bigcup_{\alpha \in A} V_{\alpha}\right)=\bigcup_{\alpha \in A} f^{-1}\left(V_{\alpha}\right) \subset \bigcup_{\alpha \in A} V_{\alpha},
$$

hence $\cup_{\alpha \in A} V_{\alpha} \in \tau_{f}$.

Now suppose that $V_{1}, V_{2} \in \tau_{f}$, which means that $f^{-1}\left(V_{1}\right) \subset V_{1}$ and $f^{-1}\left(V_{2}\right) \subset V_{2}$. Then

$$
f^{-1}\left(V_{1} \cap V_{2}\right)=f^{-1}\left(V_{1}\right) \cap f^{-1}\left(V_{2}\right) \subset V_{1} \cap V_{2},
$$

hence $V_{1} \cap V_{2} \in \tau_{f}$.

Definition. Given a set $X \neq \emptyset$ and a function $f: X \rightarrow X$, the topology $\tau_{f}$ on $X$ shown in proposition 1 is called the primal topology and $\left(X, \tau_{f}\right)$ is said to be a primal space. 
Sometimes primal spaces are called "functional Alexandroff spaces" because any primal topology $\tau_{f}$ is an Alexandroff topology, i.e. any arbitrary collection $V=\left\{V_{\alpha}\right.$ : $\alpha \in A\}$ of elements of $\tau_{f}$ we have $\cup_{\alpha \in A} V_{\alpha} \in \tau_{f}$.

We are interested in the primal topology $\tau_{\kappa}$ induced on $\mathbb{N}$ by the Collatz map. In order to work with orbits in primal spaces we use the following result by Echi and Turki [4].

Theorem 2. Let $\left(X, \tau_{f}\right)$ be a primal space. Then the following statements are equivalent:

\section{The space $\left(X, \tau_{f}\right)$ is compact.}

2. For each $x \in X$ la collection of periodic orbits is finite and $f^{r}(x)$ is in an orbit, for some $r$.

In the same line of reasoning we have another theorem by Echi which appears in [3] and requires some additional terminology. Given $A \subset X$, let us denote by $\operatorname{cl}(A)$ and $\operatorname{ker}(A)$ the closure of $A$ and the minimal open set containing $A$, respectively.

A quasi-order $\leq_{f}$ is defined on a primal space $\left(X, \tau_{f}\right)$ by $x \leq_{f} y$ if and only if $y \in \operatorname{cl}(x)$. The intervals

$$
I^{+}(x)=\left\{y \in X: x \leq_{f} y\right\} \quad \text { and } \quad I^{-}(x)=\left\{y \in X: y \leq_{f} x\right\}
$$

are precisely the sets $\operatorname{cl}(x)$ and $\operatorname{ker}(x)$, respectively.

Recall that if $f: X \rightarrow X$ is a function, a set $W \subset X$ is said to be invariant under $f$ or $f$-invariant if $f(W) \subset W$.

Definition. A set $W \subset X$ is said to be minimal of $f$ if $W$ is a minimal element in the collection of all nonempty closed invariant sets of $f$ equipped with the inclusion ordering.

Lemma 3. The minimal sets of a primal space $\left(X, \tau_{f}\right)$ are exactly the periodic orbits.

Proof. Let $A \subset X$ be a minimal subset. Then for all $x \in X$, the orbit $\Omega_{x}$ is a closed $f$-invariant set, so if $x \in A$ then $A=\Omega_{x}$ which, by the same argument, coincides with $\Omega_{f(x)}$; so $x \in \Omega_{f(x)}$, which implies that $\Omega_{x}$ is periodic. Conversely, it is clear that any periodic orbit is minimal.

By using theorem 2, Vielma and Guale [10] obtained a topological version of the Collatz conjecture. Two ingredients from topology are used in their work, the first one is defined next. 
Definition. A topological space $(X, \tau)$ is said be super-compact if there is a sub-basis such that every open cover of the $X$ from elements of the sub-basis has a sub-cover with at most two sub-basis elements.

The other basic topological ingredient is a sort of "separation axiom" introduced by Di Maio in [2].

Definition. A nontrivial topological space $(X, \tau)$ is said to be weakly $R_{0}$ if

$$
\bigcap_{x \in X} \operatorname{cl}(\{x\})=\emptyset
$$

Thus we have the next result of Vielma and Guale mentioned before.

Theorem 4. The following propositions are equivalent.

1. The Collatz conjecture is true.

2. The space $\left(\mathbb{N}, \tau_{\kappa}\right)$ is not weakly $R_{0}$.

3. The space $\left(\mathbb{N}, \tau_{\kappa}\right)$ is super-compact.

4. The space $\left(\mathbb{N}, \tau_{\kappa}\right)$ is compact and there is only one periodic orbit.

5. For any closed set $F$ in $\left(\mathbb{N}, \tau_{\kappa}\right), 1 \in F$.

\section{AN ALGEBRAIC VERSION OF THE COLLATZ CONJECTURE}

Before getting any more details about the relationship between the Collatz conjecture and the primal space $\left(\mathbb{N}, \tau_{\kappa}\right)$ we consider some special algebraic structure derived from a topology. The algebraic operations are defined in terms of operations on sets, thus we have an "addition" and a "multiplication" on open sets by using union and intersection; thus we get an algebraic structure which is slightly more basic than the one of a "ring". In general we have te following concept.

Definition. A (commutative) semiring is a nonempty set $\Lambda$ with two binary operations, called addition and multiplication, denoted by + and $\cdot$, respectively, and two different elements $\theta, \epsilon \in \Lambda$ such that the following axioms hold for all $a, b, c \in \Lambda$ :

(R1) $a+(b+c)=(a+b)+c$.

(R2) $a+\theta=\theta+a=a$. 
(R3) $a+b=b+a$.

(R4) $a \cdot(b \cdot c)=(a \cdot b) \cdot c$.

(R5) $a \cdot \epsilon=\epsilon \cdot a=a$.

(R6) $a \cdot b=b \cdot a$.

(R7) $a \cdot(b+c)=a \cdot b+a \cdot c$.

(R8) $a \cdot \theta=\theta \cdot a=\theta$.

We are interested in semirings with some very special properties which are described next.

Definition. If $\mathcal{I} \subset \Lambda$ is called an ideal of $\Lambda$ if for all $a, b \in \mathcal{I}$ and $r \in \Lambda$ we have $a+b, r a \in \mathcal{I}$. we say that an ideal $\mathcal{P}$ of $\Lambda$ is prime if $a \cdot b \in \mathcal{P}$ implies that $a \in \mathcal{P}$ or $b \in \mathcal{P}$. A semiring $\Lambda$ is said to be local if it contains a unique maximal ideal.

Thus given a topological space $(X, \tau)$, by taking the union $\cup$ as addition and the intersection $\cap$ as multiplication, $\tau$ is a semiring with $\theta=\emptyset$ and $\epsilon=X$. By using this additional algebraic structure on primal spaces Vielma and Guale [9] prove the following characterization of "the $3 x+1$ problem".

Theorem 5. The Collatz conjecture is true if and only if the primal topology $\tau_{\kappa}$ on $\mathbb{N}$ is a local semiring.

It is particularly important the fact that for any topological space $(X, \tau)$ and $F \subset X$, the set $\Phi(F)=\{V \in \tau: F \cap V=\emptyset\}$ is useful to decide whether or not the space $(X, \tau)$ is weakly $R_{0}$. Thus we have the following result.

Theorem 6. A topological space $(X, \tau)$ is not weakly $R_{0}$ if and only if there exists a closed set $F \neq \emptyset$, such that $\Phi(F)$ is the unique maximal ideal.

Proof. Suppose that $X$ is not weakly $R_{0}$ and let

$$
F=\bigcap_{x \in X} \operatorname{cl}(\{x\})
$$

then $\Phi(F)$ is a maximal ideal. Let $\mathcal{Q}$ be an ideal which is not contained in $\Phi(F)$, then there exists a $V \in \tau$ such that $V \in \mathcal{Q}$ but $V \notin \Phi(F)$, thus $V \cap F \neq \emptyset$. If $p \in V \cap F$, then $p \in \operatorname{cl}(\{x\})$ for all $x \in X$, and therefore $V=X$ which is contradiction. 
Now suppose that there is a closed set $F \neq \emptyset$ such that $\Phi(F)$ is the unique maximal ideal. Take $p \in F$ such that $p \notin \cap_{x \in X} \operatorname{cl}(\{x\})$, hence there is a $q$ with $p \notin \operatorname{cl}(\{q\})$. Then we may choose a $V \in \tau$ such that $p \in V$ but $q \notin V$. On the other hand we have that $p \in F$, therefore $V \notin \Phi(F)$ and this implies that $V=X$, which is a contradiction with the fact that $q \notin V$. Thus $F \neq \emptyset$, i.e. the space $(X, \tau)$ is not weakly $R_{0}$.

Finally we have all the ingredients to complete the proof of the Collatz conjecture's algebraic face.

Proof of theorem 5. By theorem 4 (part 2) the Collatz conjecture is true if and only if the space $\left(\mathbb{N}, \tau_{\kappa}\right)$ is not weakly $R_{0}$. By theorem 6 we have that this is equivalent to the fact that there exists a closed set $F \in \mathbb{N}$ such that $\Phi(F)$ is the unique maximal ideal. Moreover we have $F=\Omega_{1}$.

\section{A TOPOLOGICAL VERSION OF THE COLLATZ CONJECTURE}

The algebraic tool provided by theorem 5 can be used to obtain some additional properties of the primal space $\left(\mathbb{N}, \tau_{\kappa}\right)$, which combines with the following result from [7] to give us a new equivalence with the Collatz conjecture.

Lemma 7. Let $\left(X, \tau_{f}\right)$ be a primal space and let $p \in X$. The connected component of $p$ es the set

$$
\left\{y: p \in \Omega_{x, f} \text { whenever } x \in \Omega_{y, f}\right\} \cup\left\{y: x \in \Omega_{y, f} \text { whenever } x \in \Omega_{p, f}\right\} \text {. }
$$

Theorem 8. The Collatz conjecture is true if and only if the primal space $\left(\mathbb{N}, \tau_{\kappa}\right)$ is connected.

Proof. Suppose that the Collatz conjecture holds, then by part 4 of theorem 4, the space $\left(\mathbb{N}, \tau_{\kappa}\right)$ is compact and $\Omega_{1}$ is the only one periodic orbit, thus the connected component containing 1 is $\mathbb{N}$ and therefore, by lemma 7 , the space $\left(\mathbb{N}, \tau_{\kappa}\right)$ is connected.

On the other hand, if we suppose that the space $\left(\mathbb{N}, \tau_{\kappa}\right)$ is connected we have that it is a terminal connected primal space, because $\Omega_{1}$ has finite closure. Then, by theorem 4 (part 3), the space $\left(\mathbb{N}, \tau_{\kappa}\right)$ is supercompact which implies the Collatz conjecture.

Let us note that any open cover of the primal space $\left(\mathbb{N}, \tau_{\kappa}\right)$ must contain $\mathbb{N}$ since this is the only open set which contains 1 , a condition from proposition 4 in theorem 4 can be dropped thus we have the following consequence of theorem 8.

Corollary 9. If the Collatz conjecture is true then the primal space $\left(\mathbb{N}, \tau_{\kappa}\right)$ is compact. 
By using theorem 8 we are able to investigate some other topological $\left(\mathbb{N}, \tau_{\kappa}\right)$ using curves in $\mathbb{N}$. First recall some basic terminology.

Definition. A path in a topological space $(X, \tau)$ is a continuous map $\gamma:[0,1] \rightarrow X$, where the topological structure of $[0,1]$ is the one induced by the Euclidean topology of the real numbers $\mathbb{R}$. A path $\gamma$ is based at $p \in X$ if $\gamma(0)=p$. A loop in $X$ is path $\gamma$ such that $\gamma(0)=\gamma(1)$. The space is said to be path-connected if for every $p, q \in X$ there is a path $\gamma$ in $X$ such that $\gamma(0)=p$ and $\gamma(1)=q$.

Theorem 10. The primal space $\left(\mathbb{N}, \tau_{\kappa}\right)$ is connected if and only if there exists a loop $\gamma$ based at 1 such that the image of $\gamma$ is $\mathbb{N}$.

Proof. Suppose that the primal space $\left(\mathbb{N}, \tau_{\kappa}\right)$ is connected and consider the intervals $I_{n}=\left(a_{n}, b_{n}\right) \cap[0,1]$ (which are open in $[0,1]$ ) where

$$
a_{n}=\frac{2 n+1}{2 n(n+1)} \quad \text { and } \quad b_{n}=\frac{2 n+3}{2 n(n+1)} .
$$

Define $\gamma:[0,1] \rightarrow \mathbb{N}$ by

$$
\gamma(t)= \begin{cases}n & \text { if } t \in I_{n} \\ 1 & \text { otherwise }\end{cases}
$$

If $O \in \tau_{\kappa}$ and $1 \notin O$; let $k=\min O$, then

$$
\gamma^{-1}(O)=\bigcup_{n \geq k} I_{k}
$$

which is open. On the other hand if $1 \in O$ we have $O=\mathbb{N}$, then and $\gamma^{-1}(O)=[0,1]$. Therefore $\gamma$ is continuous on $[0,1]$ and $\gamma([0,1])=\mathbb{N}$.

Conversely, if we suppose that $\gamma$ is a loop in $\mathbb{N}$ based at 1 with $\gamma([0,1])=\mathbb{N}$, then the space $\left(\mathbb{N}, \tau_{\kappa}\right)$ is paht-connected connected, and therefore it is connected.

Finally we have the following results.

Corollary 11. The Collatz conjecture is true if and only if there exists a loop $\gamma$ based at 1 such that image of $\gamma$ is $\mathbb{N}$.

Corollary 12. The Collatz conjecture is true if and only if $\left(\mathbb{N}, \tau_{\kappa}\right)$ is path-connected.

\section{ACKNOWLEDGEMENT}

This work was supported by Decanato de Investigación de la Escuela Superior Politécnica del Litoral, project FCNM-08-2017 


\section{REFERENCES}

[1] Daniel J. Bernstein and Jeffery C. Lagarias, The 3x +1 conjugacy map, Canad. $J$. Math. 48 (1996) 1154-1169.

[2] Giuseppe Di Maio, A separation axiom weaker than $R_{0}$, Indian J. Pure Appl. Math. 16 (1985) 373-375.

[3] Othman Echi, The categories of ows of Set and Top, Topology Appl. 159 (2012) 2357-2366.

[4] Othman Echi and Tarek Turki, Spectral primal spaces, J. Algebra Appl. 18 (2019) $1-12$.

[5] Michal Jerzy Kukiela, On homotopy types of Alexandroff spaces, Order 27 (2010) 9-21.

[6] Jeffrey C. Lagarias, The $3 x+1$ problem and its generalizations, Amer. Math. Monthly 44 (1985) 3-22.

[7] Fatemah Ayatollah Zadeh Shirazi and Nasser Golestani, Functional Alexandroff spaces, Hacet. J. Math. Stat. 40 (2011) 515-522.

[8] Terence Tao, Almost all orbits of the Collatz map attain almost bounded values. arXiv:1909:03562v2, 2019.

[9] Jorge Vielma and Ángel Guale, The algebraic face of the Collatz conjecture, Preprint (2019).

[10] Jorge Vielma and Ángel Guale, A topological approach to the Ulam-Kakutani-Collatz conjecture, Topology Appl. 256 (2019) $1-6$.

[11] Jorge Vielma, Ángel Guale, and Fernando Mejías, Paths in primal spaces and the Collatz conjecture, Quaest. Math. 43 (2020) 1-7. 